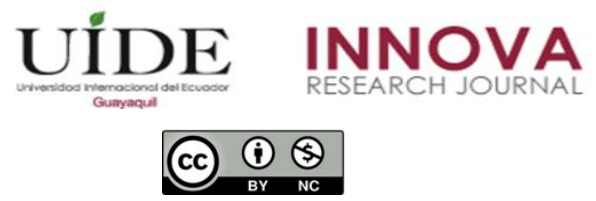

INNOVA Research Journal, ISSN 2477-9024

(Septiembre-Diciembre 2020). Vol. 5, No.3.1 pp. 40-57

DOI: https://doi.org/10.33890/innova.v5.n3.1.2020.1540

URL: http://revistas.uide.edu.ec/index.php/innova/index

Correo: innova@uide.edu.ec

\title{
Estandarización del Cuestionario de Actitudes y Estrategias Cognitivas Sociales en Escolares de Instituciones Educativas del Distrito de Ate
}

\section{Standardization of the Questionnaire of Attitudes and Social Cognitive Strategies in Schoolchildren of Educational Institutions of the Ate district}

\author{
Edith Honorina Jara Ames \\ (O) https://orcid.org/0000-0002-4180-9418 \\ Taniht Lisseth Cubas Romero \\ (D) https://orcid.org/0000-0002-7988-3285 \\ Roxana Maribel Cárdenas Vila \\ (C) https://orcid.org/0000-0002-1089-6299 \\ Omar Arturo Lizarraga Carrasco \\ (O) https://orcid.org/0000-0002-5591-9300 \\ Universidad César Vallejo, Perú
}

Autor para correspondencia: ejaraames@hotmail.com; tanihtcubas@ gmail.com;

roxanamaribelcv@hotmail.com; arturo0781@hotmail.com

Fecha de recepción: 16 de septiembre de 2020 - Fecha de aceptación: 20 de noviembre de 2020

\section{Resumen}

La investigación tuvo como finalidad evaluar las propiedades psicométricas del Cuestionario de Actitudes y Estrategias Cognitivas Sociales (AECS) en escolares de instituciones educativas del Distrito de Ate. Para ello se trabajó un estudio de diseño no experimental de tipo instrumental con una muestra de 979 estudiantes de ambos sexos entre las edades de 12 a 17 años. Para la obtención del resultado se empleará la estadística descriptiva, a través de la media y la desviación estándar. También se utilizará la estadística inferencial analizando la confiabilidad por medio del coeficiente Alpha de Crombach, la validez del constructo desde el análisis factorial y los baremos normativos que se presentarán en percentiles. El diseño utilizado en esta investigación fue No experimental de tipo instrumental. Se empleó el instrumento de actitudes y estrategias cognitivas sociales el cual consta de 137 preguntas con una escala múltiple del 1 al 7. De acuerdo con los resultados estadísticos se concluye que el test es válido y confiable para la población de escolares del Distrito de Ate, además se elaboraron los percentiles correspondientes.

Palabras claves: Estrategias cognitivas sociales; actitudes sociales; estandarización; competencia social; adolescentes.

\footnotetext{
Abstract

The purpose of the research was to evaluate the psychometric properties of the Attitudes and Social Cognitive Strategies Questionnaire (AECS) in schoolchildren from educational institutions in the 
District of Ate. For this, a study of non-experimental design of an instrumental type was carried out with a sample of 979 students of both sexes between the ages of 12 to 17 years. Descriptive statistics will be used to obtain the result, through the mean and standard deviation. Inferential statistics will also be used, analyzing the reliability by means of Crombach's Alpha coefficient, the validity of the construct from the factor analysis and the normative scales that will be presented in percentiles. The design used in this research was non-experimental of the instrumental type. The instrument of attitudes and social cognitive strategies was used, which consists of 137 questions with a multiple scale from 1 to 7 . According to the statistical results, it is concluded that the test is valid and reliable for the population of schoolchildren in the Ate District. In addition, the corresponding percentiles were elaborated.

Keywords: Social cognitive strategies; social attitudes; standardization; social competence; adolescents.

\section{Introducción}

El incremento de los problemas en la adolescencia se vincula con sus competencias interpersonales, esto como resultado de los cambios sociales, culturales e institucionales que ocurren en el interior de la dinámica de los principales agentes socializadores, la familia, la escuela y la sociedad. En sociedades como la peruana que ha experimentado cambios sociales, políticos, económicos vertiginosos, en un contexto de interculturalidad y de la trasformación tecnológica los adolescentes se han visto en la necesidad de interiorizar nuevas formas de relacionarse entre sí, exigiéndoles un nivel de entendimiento con el propósito de una lograr una convivencia pacífica y empática.

Según Plunkett (2000), refiere que la familia, la escuela y el entorno perfilan la capacidad de interacción de los seres humanos acorde al entorno en el que se desarrolla cotidianamente, cuando estos espacios se alteran y son inestables, los individuos suelen adoptar comportamientos, cogniciones y emociones según su realidad interactiva, esta se convierte en un escenario dañino creando grandes conflictos que repercuten en su desarrollo y bienestar. Esta situación coadyuva a producir tensión, frustración y malestar a las personas, más aún si son adolescentes.

Por ello, la competencia social adecuada permite que el adolescente se vincule exitosamente con logros sociales y académicos, denote un ajuste personal y social que contribuya con su bienestar psicoemocional, generando aceptación. La competencia social definida por Moraleda, González, y García (2004), como la capacidad que tiene los individuos de lograr desarrollarse de manera emocional y actitudinal frente a situaciones diversas. Esta competencia social posibilita que los adolescentes desarrollen un conjunto de habilidades afectivas y sociales que les permita adecuarse exitosamente a los diferentes ámbitos interpersonales principalmente las que se dan en la escuela obteniendo aceptación y gratificación de sus pares (Betina y Contini, 2011).

La competencia social permite que los individuos se desenvuelvan favorablemente con su entorno. Este ajuste personal y social es la consecuencia de tener y poner en práctica un conjunto de comportamientos que son positivas y aceptadas social y culturalmente como: la tolerancia, autonomía, solidaridad, respetuosos de las reglas, normas y los valores sociales. Castillo y 
Eugenio, (2019). El fracaso o éxito de la relación que se da entre los adolescentes depende, de la capacidad social que posee, la cual comprende habilidades y capacidades sociales y afectivas que favorecen su adaptación y desarrollo en los diferentes ambientes en las que se desenvuelven.

Por otro lado, la incompetencia social. se relaciona con baja aceptación o rechazo por parte de los pares, rendimiento por debajo del promedio, ausentismo o abandono del sistema escolar, problemas en sus transacciones sociales, baja autoestima, desajustes psicológicos, inadaptación y vulnerabilidad (Del Prette, Z. A. - Del Prette, A., 2002). Esto evidencia que varios de los adolescentes tienen conductas inadecuadas que denotan la inexperiencia de afrontar situaciones de presión que surgen en la vida cotidiana. Como lo sugiere (Santos, 2015), el desarrollo de habilidades y estrategias utilizadas en la resolución de problemas sociales cotidianas garantizan la adaptación, y la inhabilidad se relaciona con la inadaptación. En ese sentido es importante aprender y desempeñar un conjunto de comportamientos que se adapten a escenarios conflictivos, que permitan alcanzar metas utilizando conocimientos y respetando normas y reglas.

La interacción social que se genera en las instituciones educativas contribuye en el progreso académico y convivencia, estos son aspectos importantes para la comunicación y el respeto hacia los otros; además permiten incrementar el rendimiento académico, así como la empatía, el aprendizaje cooperativo, la motivación escolar y la participación de los educandos en el proceso educativo (López, Soto, Carbajal y Nel, 2013). El contexto escolar es el escenario idóneo para desarrollar y poner en práctica estrategias y habilidades que contribuyan con la adaptación social. Estas habilidades se aprenden y se practican desde muy temprana edad, favorecen con mejorar la autonomía, la aceptación de las reglas y normas sociales, garantizando una mejor comunicación entre pares y adultos, lo referido es muy relevante si se contempla que la competencia social que se desarrolla desde la infancia está vinculada con la capacidad de adaptación social, escolar y psicoemocional en la adolescencia y por ende en la vida adulta de los individuos (Bravo y Herrera, 2011).

En este sentido la competencia social se relaciona con el logro de un desenvolvimiento socialmente adecuado, cuando esta situación no se logra se habla de términos como retraimiento social, agresividad social y aislamiento (Rangel, 2015). Manifestaciones como la agresión, la intransigencia social y el liderazgo nocivo se evidencia en contextos educativos en la cual el adolescente no sabe o le cuesta manejar su comportamiento impulsivo. Para afrontar adecuadamente esta realidad, desde un marco pedagógico integral es necesario armonizar aspectos académicos, sociales y afectivos, que involucra la formación de los educandos promoviendo su participación más activa con su entorno (Fernández, Luquez y Leal, 2010).

Cabe señalar que la interacción social en los niños y adolescentes se da principalmente en dos ámbitos: el familiar y el fuera del hogar. Para Perinat (1998), significa que estar fuera de la familia tiene dos trayectorias, la 'vertical' las relaciones jerárquicas que se dan en la escuela, que se establecen entre profesores y estudiantes; y la 'horizontal', las que contemplan las relaciones entre pares, las mismas que se forman entre compañeros y amigos fuera del ambiente escolar.

Ante ello, surge la preocupación de los docentes que trabajan con adolescentes en que estos logren competencias sociales que les permita enfrentarse a las diversas demandas de su 
entorno. Sin embargo, el aumento de la violencia familiar, en el ámbito escolar, en la sociedad, se da primordialmente en los adolescentes en los diferentes estratos sociales y con mayor frecuencia se dan delitos graves. Con las nuevas tecnologías de comunicación se ha acrecentado el aislamiento social, y ha disminuido el contacto con los demás Ello se traduce también en la dificultad que presentan para resolver conflictos, socializar asertivamente con sus pares, además de incrementarse el uso de un lenguaje soez e indecente.

Actualmente, en el contexto educativo peruano la competencia social se vuelve indispensable en el desempeño conductual de los estudiantes en los diferentes niveles de forma que aquellos que presenten habilidades sociales positivas se desempeñan mucho mejor en el ámbito escolar, social y emocional. En ese sentido la enseñanza de la competencia social se convierte en requisito fundamental para la buena adaptación del estudiante, existiendo una estrecha relación con los aprendizajes y el desarrollo cognitivo.

Según Moraleda (Citado por Villena, D. 2015), la labor del docente como animador de la clase deberá ser el aprender a discriminar qué tipo de roles necesita cada grupo en cada momento y ayudar a los miembros por a intervenir en este conocimiento. Al mismo tiempo, ayudarles a desempeñar estos roles requeridos, de modo que el grupo pueda alcanzar así un mejor funcionamiento.

Para López, Garrido, Rodríguez y Paíno (2002) las personas experimentan la necesidad de aprender a nivel del pensamiento inferencial y crítico ya que esto les permitirá ser competentes en las diversas asignaturas y experiencias sociales en la etapa escolar. Para ello, existen diversos escenarios en la cual se logran mejorar la competencia social. Una de ellas es la intervención educativa incrementa el desarrollo de la competencia social y se ajusta a los diferentes tipos de estudiantes y no sólo para los que manifiestan alguna dificultad (MartínezOtero, 2001).

Por esas consideraciones se pone en relevancia que el aprendizaje de la competencia social como fundamento de esta investigación se sustenta por un lado en la teoría cognitiva social del aprendizaje de Bandura, haciendo referencia que la mayor parte del comportamiento humano se aprende a través de la observación de modelos, produciendo cambios en el observador desde el aprendizaje de capacidades cognitivas nuevas, inhibición o desinhibición de conductas ya aprendidas, la evocación de recordar respuestas, la generalización de conductas, la capacidad de activar emociones en una situación empática (Ruiz, 2017).

Y, por otro lado, se ha considerado también al modelo teórico de carácter integrador desarrollado por Moraleda, Gonzales y García-Gallo (1998), la misma que concibe que la competencia social reúne variables actitudinales y cognitivas. Tales variables, son una fuente enriquecedora para los adolescentes, que permite el éxito o el fracaso en las relaciones con los demás; estas actitudes y estrategias cognitivas sociales permiten que los individuos con una elevada competencia social denoten un mayor grado de satisfacción personal con el entorno en el que se despliegan consigo mismas, además de ser socialmente más aceptadas por los demás (Lorente, 2014). 
Cabe señalar que las instituciones educativas del distrito de Ate, albergan alumnos de secundaria, quienes en su mayoría reflejan no poseer competencias interpersonales suficientes para solucionar dificultades; los maestros, han observado ciertas características asociadas con dificultades para socializar entre ellos, conformistas, renuentes a acatar reglas y normas, poco empáticos y no asumen responsabilidades a partir de las decisiones que toman. Esta realidad se torna prejudicial para su desarrollo personal, social y emocional.

Es por ello que surge el interés de estandarizar el instrumento psicológico denominando Cuestionario de Actitudes y Estrategias Cognitivas Sociales (AESC) en alumnos de secundaria y ser utilizado en las escuelas del distrito de Ate. Que contribuya a que los adolescentes del nivel secundario reflexionen y aprendan sobre las estrategias cognitivas de pensamiento y la competencia social. El modelo para trabajar emplea tres variables la cuales son: actitudinales, cognitivas y procedimentales que esté relacionado con la competencia social en los escolares la cual se busca como función principal desarrollar y potenciar esta habilidad.

Las transacciones sociales de los adolescentes dependen principalmente de poseer ciertas variables actitudinales y cognitivas consistentes y estables a través del tiempo y que constituyen con su competencia o incompetencia social. Estas actitudes sociales contemplan lo que ellos piensan, sienten, y la forma de cómo actúan con su entorno sociocultural. Las variables relacionadas con las actitudes sociales presentan tres factores: prosocial o social, hace hincapié al factor facilitador de las relaciones, el asocial que es el inhibidor en las relaciones y un tercer factor que es antisocial, que concentran dos aspectos de orden menor: que es la dominancia, la cual tiene como tendencia a buscar dominar a los demás a través del poder, así como también a competir y ser superior a los demás y el segundo, es la agresión terquedad, lo cual presentan como la tendencia a la manifestación agresiva a los demás, hostilidad, tendencia rígida, y finalmente la envidia o tristeza por el bien ajeno (Castillo, 2014).

Por otro lado, se contempla las variables cognitivas ligadas al éxito o fracaso de los adolescentes y su relación social esta se vincula con tres factores: Percepción social, estilos y estrategias cognitivas, las mismas que les permiten a los adolescentes resolver con eficacia o no problemas sociales. (Moraleda, citado por Castillo. M y Eugenio, D. 2019).

En sintonía con lo manifestado por los autores la competencia social se relaciona con la expresión de ciertas actitudes sociales básicas y por otro lado se le pone mayor atención en como ellos procesa, almacena y aplica la información en su interacción (cognición social). Lo cierto es que la competencia social ayuda en este caso a los adolescentes a desarrollarse en aquellas áreas que se vinculan con un adecuado ajuste personal y social.

El Cuestionario de Actitudes y Estrategias Cognitivas Sociales es un instrumento de valoración que ofrece una visión de las competencias sociales en los adolescentes, es decir evaluar las variables que son factibles o que dificultan la interacción social, evaluando aspectos relacionados con el respeto de normas, comprensión de los demás, capacidad de compartir, seguridad de sí mismo, liderazgo, tendencias agresivas, grado de dominancia, tendencia al aislamiento, etc. Igualmente evalúa las variables cognitivas indispensables para resolver eficientemente una situación problemática que demanda capacidad de observación y análisis de la situación social evaluando posibles alternativas. 
El Cuestionario AECS evalúa 9 actitudes sociales y 10 estrategias de pensamiento social en estudiantes adolescentes de ambos sexos entre las edades de 12 y 17 años. La prueba psicológica cuenta con dos escalas la primera es de Actitud Social la cual comprende la forma de proceder de los adolescentes en la interacción con sus pares, y la segunda es la escala de Pensamiento Social, en donde contempla las estrategias y habilidades que el adolescente toma como recurso en el entorno donde se encuentra, en la cual asume decisiones en sus distintas situaciones diarias. En el aspecto cognitivo, sus logros o frustraciones en sus relaciones interpersonales se sustentan en tres principales factores: el primero se relaciona con la percepción social, el segundo con sus estilos cognitivos y el último son las estrategias cognitivas la cual se emplea en la resolución de problemas sociales (Moraleda et al., 2004).

En la elaboración inicial de las escalas del cuestionario en mención se siguieron tres pasos: el primero fue el procedimiento selectivo de jueces, se diseñaron 19 escalas tipo Likert compuesta de 30 ítems cada una. Desde su evaluación por criterio jueces realizaron una primera selección de ítem, en donde fue reducido a 10 ítems por escala. El segundo paso consistió en aplicar los protocolos en una muestra piloto, para verificar la fiabilidad y homogeneidad de los ítems, eliminando algunos y mejorando la redacción de otros. Finalmente se aplicó el nuevo cuestionario a una muestra representativa de la población, procediendo a confeccionar los baremos y obtener puntajes definitivos (Moraleda, Gonzales y García, 1998).

Las escalas de actitud social son: Conformidad con lo que es socialmente correcto (Con), Sensibilidad Social (Sen), Ayuda y Colaboración (Ac), Seguridad y Firmeza en la interacción (Sf), Liderazgo Prosocial (Lid), Agresividad-Terquedad (Agr), Dominancia (Dom), ApatíaRetraimiento (Ap), Ansiedad-Timidez (Ans). Las escalas de pensamiento social son: Independencia frente a Dependencia de Campo (Ind), Convergencia frente a Divergencia (Cv), Percepción y Expectativas sobre la relación social (Per), Percepción por el sujeto del modo de ejercer sus padres la autoridad en el hogar (Dem), Percepción por el sujeto de la calidad de aceptación y acogida que recibe de sus padres (Hos), Habilidad en la observación y retención de la información relevante sobre las situaciones sociales (Obs), Habilidad en la búsqueda de soluciones alternativas para resolver los problemas sociales (Alt), Habilidad para anticipar y comprender las consecuencias que posiblemente se seguirán de los comportamientos sociales (Cons), Habilidad para elegir los medios adecuados a los fines que se persiguen en el comportamiento social (Med).

En el contexto peruano el instrumento AECS que se ha utilizado, será de acuerdo con la validación realizada en la investigación "Propiedades Psicométricas del Cuestionario de Actitudes y Estrategias cognitivas Sociales en alumnos de secundaria" por Polo (2014). Para validar el instrumento la autora utilizó una muestra de 329 adolescentes entre los 12 y 17 años y fue realizada en dos instituciones educativas de la ciudad de Trujillo. En su estudio realizó la validez de constructo mediante el Coeficiente de Correlación ítem-test corregida, en la cual se eliminaron 12 ítems; obteniendo puntuaciones en las escalas de actitud social valores que oscilan entre .191 y .481; y en las escalas de pensamiento social se eliminaron 8 ítems, donde los valores oscilan entre .291 y .591 . Asimismo, tuvo una confiabilidad en donde los valores de las escalas del instrumento oscilan entre .487 y .718 donde alcanzaron niveles entre moderados, mínimamente aceptable y respetable. 


\section{Materiales y métodos}

\section{Método}

La investigación tuvo una metodología con enfoque cuantitativo y un diseño de tipo no experimental con corte transversal ya que no se manipulo ni se sometió a prueba la variable de estudio (Hernández, Fernández y Baptista, 2014).

Para comprobar empíricamente las garantías psicométricas de las pruebas que se expresan como instrumento de medición, se fundamenta principalmente en la confirmación práctica del contenido fidedigno en el cual evalúa (validez) y en su exactitud (fiabilidad). Estos factores están íntimamente ligados, es decir un instrumento no puede ser válido si no es fiable al mismo tiempo. Es importante mencionar que las propiedades psicométricas de las pruebas constituyen bondades que da una mayor garantía científica la cual aporta la medición que se efectúa desde la psicología (Rodríguez y Morelio, 2012)

\section{Población y muestra}

La investigación estuvo constituida por 11775 estudiantes adolescentes de ambos sexos, del primer al quinto año de secundaria, sus edades oscilaron entre 12 y 17 años de 15 instituciones educativas del distrito de Ate Vitarte.

Teniendo en cuenta que la cantidad de la población que fue 11775 de las instituciones elegidas para el estudio. El tamaño de la muestra ha sido delimitado por el proceso probabilísticos mediante la fórmula estadística de poblaciones infinitas con un nivel de confianza del $95 \%$ con un margen de error del 5\%. La misma que equivale a 979 estudiantes.

\section{Instrumento:}

El Cuestionario de Actitudes y Estrategias Cognitivas Sociales (AESC) tiene como autores a M. Moraleda, A. González Galán y J. García -Gallo. Donde su aplicación puede tomar de manera colectiva e individual en adolescentes de 12 a 17 años, se toma 30 minutos aproximadamente para el llenado de respuestas. En donde su finalidad es la evaluación de 9 actitudes sociales y 10 estrategias de pensamiento social. El material que se utiliza para la evaluación es el manual, el cuadernillo, la hoja de respuesta y la descripción de resultados.

Para su calificación sus puntuaciones son de manera directa en la cual se puede obtener de la misma hoja de respuestas. La puntación directa de cada escala se obtiene a través de la suma de sus respuestas de cada elemento de la escala, en una segunda hoja se suma las respuestas según el orden que se indica y se anota la puntuación directa de cada escala luego estas puntuaciones directas se pueden transformar en percentiles consultando lo que propone el manual (Moraleda et al., 2004, p.48.).

Para la interpretación del instrumento se hace una valoración de cada escala según su puntuación de percentiles y analizar sus rangos dentro de la estructura de la competencia social 
de cada persona. Es importante tener en cuenta que las puntuaciones altas se dan (Pc $>85)$ y las puntaciones bajas (Pc <15). Además, se debe elegir a aquellas actitudes y comportamiento descriptivos más relevante de cada una de las escalas.

\section{Análisis estadísticos}

En el estudio dentro del análisis cuantitativo realizado en el Paquete Estadístico para la Ciencias Sociales (SPSS) versión 25, se procedió a codificar los datos obtenidos, luego se realizó el Alfa de Cronbach para para el instrumento permitiendo verificar la fiabilidad, además se utilizó el estadístico Kolgomorov Smirnov, para determinar si los datos son paramétricos o no paramétricos y así se pudo utilizar el estadístico adecuado. A partir de ahí se trabajó la validez de constructo a través del KMO, la prueba de Bartlett, análisis de comunalidades. Asimismo, se procedió con la baremación para luego realizar los percentiles de cada escala.

\section{Resultados}

\section{Validez de constructo}

Se realizó el análisis del KMO la cual tuvo (.827) es decir es mayor que 0.5 lo que indica que es aceptable para el análisis factorial ya que mientras esté más cerca al 1 es mejor. Asimismo, en la prueba de esfericidad de Bartlett (.000) indica, es menor que 0.05 la cual es indicado el análisis factorial.

\section{Tabla 1}

Prueba de KMO y Bartlett

\begin{tabular}{lll}
\hline Prueba de KMO y Bartlett & & \\
\hline Medida Kaiser-Meyer-Olkin de adecuación de muestreo & .827 \\
Prueba de esfericidad de Bartlett & Aprox. Chi-cuadrado & 112712.710 \\
& gl & 9316 \\
& Sig. & .000 \\
\hline
\end{tabular}

Fuente: elaboración propia

En cuanto a la tabla 2 en el análisis de comunalidad de las escalas de actitud social; se obtuvo que el ítem 10 presenta el valor por debajo del criterio establecido $\geq 0.30$. 


\section{Tabla 2}

Análisis de Comunalidades de las Escalas de Actitud Social

\begin{tabular}{|c|c|c|c|c|c|c|c|}
\hline \multicolumn{8}{|c|}{ Análisis de comunalidades de Actitud Social } \\
\hline \multirow{2}{*}{ CONS } & & \multicolumn{2}{|c|}{ SEN } & \multicolumn{2}{|l|}{$\mathbf{A C}$} & \multicolumn{2}{|l|}{ SF } \\
\hline & $\overline{\mathbf{h}^{2}}$ & & $\mathbf{h}^{2}$ & & $\mathbf{h}^{2}$ & & $\mathbf{h}^{2}$ \\
\hline Ítem 8 & .767 & Ítem 1 & .411 & Ítem 2 & .441 & Ítem 4 & .536 \\
\hline Ítem 14 & .702 & Ítem 15 & .431 & Ítem 9 & .574 & Ítem 11 & .367 \\
\hline Ítem 22 & .469 & Ítem 23 & .350 & Ítem 16 & .588 & Ítem 18 & .490 \\
\hline Ítem 29 & .601 & Ítem 30 & .468 & Ítem 24 & .441 & Ítem 26 & .372 \\
\hline Ítem 37 & .675 & Ítem 44 & .812 & Ítem 31 & .474 & Ítem 33 & .530 \\
\hline Ítem 43 & .752 & Ítem 50 & .525 & Ítem 38 & .601 & Ítem 39 & .579 \\
\hline Ítem 49 & .661 & Ítem 58 & .480 & Ítem 45 & .792 & Ítem 46 & .603 \\
\hline Ítem 57 & .549 & Ítem 66 & .526 & Ítem 51 & .552 & Ítem 53 & .550 \\
\hline \multirow[t]{2}{*}{ Ítem 65} & .763 & & & Ítem 59 & .542 & Ítem 61 & .593 \\
\hline & & & & Ítem 67 & .695 & Ítem 69 & .622 \\
\hline \multirow[t]{2}{*}{ AGR } & & DOM & & $\mathbf{A P}$ & & ANS & \\
\hline & $\mathbf{h}^{2}$ & & $\mathbf{h}^{2}$ & & $\mathbf{h}^{2}$ & & $\mathbf{h}^{2}$ \\
\hline Ítem 5 & .679 & Ítem 32 & .473 & Ítem 6 & .415 & Ítem 7 & .662 \\
\hline Ítem 12 & .618 & Ítem 40 & .400 & Ítem 13 & .551 & Ítem 21 & .521 \\
\hline Ítem 19 & .697 & Ítem 52 & .575 & Ítem 20 & .450 & Ítem 36 & .472 \\
\hline Ítem 27 & .625 & Ítem 54 & .636 & Ítem 28 & .338 & Ítem 42 & .482 \\
\hline Ítem 34 & .499 & Ítem 60 & .621 & Ítem 35 & .395 & Ítem 48 & .661 \\
\hline Ítem 47 & .808 & Ítem 68 & .563 & Ítem 41 & .496 & Ítem 56 & .528 \\
\hline Ítem 62 & .688 & & & Ítem 55 & .466 & Ítem 65 & .321 \\
\hline Ítem 70 & .570 & & & & & & \\
\hline \multicolumn{8}{|c|}{ LID } \\
\hline & $\overline{\mathbf{h}^{2}}$ & & & & & & \\
\hline Ítem 3 & .603 & & & & & & \\
\hline Ítem 10 & .216 & & & & & & \\
\hline Ítem 17 & .646 & & & & & & \\
\hline Ítem 25 & .649 & & & & & & \\
\hline
\end{tabular}

Fuente: elaboración propia

En la tabla 3 en el análisis de comunalidad; se obtuvo que los ítems 133 y 135 presentan valores por debajo del criterio establecido $\geq 0.30$.

\section{Tabla 3}

Análisis de Comunalidades de las Escalas de Pensamiento Social (h2 = comunalidad)

\begin{tabular}{|c|c|c|c|c|c|c|c|}
\hline \multirow[t]{2}{*}{ IMP } & \multicolumn{3}{|c|}{ IND } & \multicolumn{2}{|l|}{ CV } & \multicolumn{2}{|l|}{ PER } \\
\hline & $h^{2}$ & & $h^{2}$ & & $\mathbf{h}^{2}$ & & $\mathbf{h}^{2}$ \\
\hline Ítem 73 & .739 & Ítem 76 & .398 & Ítem 72 & .727 & Ítem 91 & .473 \\
\hline Ítem 75 & .481 & Ítem 79 & .642 & Ítem 74 & .528 & Ítem 94 & .430 \\
\hline Ítem 78 & .655 & Ítem 82 & .583 & Ítem 77 & .701 & Ítem 96 & .589 \\
\hline Ítem 81 & .616 & Ítem 84 & .660 & Ítem 80 & 638 & Ítem 98 & 456 \\
\hline Ítem 83 & .457 & Ítem 86 & .569 & Ítem 87 & 387 & Ítem 102 & 419 \\
\hline Ítem 85 & .607 & Ítem 89 & .618 & & & & \\
\hline Ítem 88 & .321 & & & & & & \\
\hline
\end{tabular}




\begin{tabular}{|c|c|c|c|c|c|c|c|}
\hline \multicolumn{2}{|c|}{ OBS } & \multicolumn{2}{|l|}{ ALT } & \multicolumn{2}{|l|}{ CONS } & \multicolumn{2}{|l|}{ MED } \\
\hline & $\mathbf{h}^{2}$ & & $\mathbf{h}^{2}$ & & $\mathbf{h}^{2}$ & & $\mathbf{h}^{2}$ \\
\hline Ítem 106 & .315 & Ítem 107 & .217 & Ítem 108 & .408 & Ítem 109 & .353 \\
\hline Ítem 110 & .560 & Ítem 111 & .509 & Ítem 112 & .567 & Ítem 113 & .599 \\
\hline Ítem 114 & .330 & Ítem 115 & .554 & Ítem 116 & .568 & Ítem 117 & .499 \\
\hline Ítem 118 & .750 & Ítem 119 & .546 & Ítem 120 & .384 & Ítem 121 & .483 \\
\hline Item 122 & .591 & Ítem 123 & .541 & Ítem 124 & .568 & Item 125 & .529 \\
\hline Ítem 126 & .597 & Ítem 127 & .427 & Ítem 128 & .448 & Ítem 129 & .457 \\
\hline Ítem 130 & .577 & Ítem 131 & .371 & Ítem 132 & .719 & Ítem 133 & .222 \\
\hline Ítem 134 & .507 & Ítem 135 & .267 & Ítem 136 & .581 & Ítem 137 & .518 \\
\hline DEM & & HOS & & & & & \\
\hline & $\mathbf{h}^{2}$ & & $\mathbf{h}^{2}$ & & & & \\
\hline Ítem 90 & .359 & & & & & & \\
\hline Ítem 92 & .565 & & & & & & \\
\hline Ítem 93 & .639 & & & & & & \\
\hline Ítem 95 & .629 & & & & & & \\
\hline Ítem 97 & .580 & & & & & & \\
\hline
\end{tabular}

Fuente: elaboración propia

En la tabla 4 se observa que los valores de confiabilidad oscilan entre 0.481 y 0.741 , indicando que las escalas de Sensibilidad Social (Sen), Ayuda y colaboración (Ac), Seguridad y firmeza (Sf), Liderazgo prosocial (Lid), Dominancia (Dom), Ansiedad-timidez (Ans) y Agresividad-terquedad (Agr) alcanzaron niveles de confiabilidad alto; no obstante, Conformidad (Con) y Apatía-retraimiento (Ap) se encuentran en el nivel medio.

\section{Tabla 4}

Confiabilidad de las escalas de actitud social - Cuestionario de AECS

\begin{tabular}{ll}
\hline ESCALA & Alfa de Cronbach \\
\hline CON & .557 \\
SEN & .739 \\
AC & .741 \\
SF & .696 \\
LID & .690 \\
AGR & .674 \\
DOM & .648 \\
AP & .481 \\
ANS & .624 \\
\hline
\end{tabular}

Fuente: elaboración propia

En la tabla 5 se observa que los valores de confiabilidad oscilan entre 0.612 y 0.827 , indicando que todas las escalas alcanzaron niveles de confiabilidad alto. (Palella y Martins, 2012, p.169). 


\section{Tabla 5}

Confiabilidad de las escalas de pensamiento social - Cuestionario de AECS

\begin{tabular}{ll}
\hline ESCALA & Alfa de Cronbach \\
\hline IMP & .753 \\
CV & .612 \\
PER & .718 \\
DEM & .724 \\
IND & .694 \\
HOS & .797 \\
OBS & .768 \\
ALT & .802 \\
CONS & .776 \\
MED & .827 \\
\hline
\end{tabular}

Fuente: elaboración propia

\section{Baremación}

Para la baremación, se procedió a realizar la prueba de normalidad, donde se trabajó con el Kolmogorov-Smirnova (K-S) para determinar si la prueba es paramétrica $(\mathrm{p}>0.05)$ o no paramétrica $(\mathrm{p}<0.05)$. En la tabla 6 , se halló que no se distribuye de forma normal $(\mathrm{p}<0.05)$, por lo tanto, se comprueba el empleo de estadísticos no paramétricos por tanto se utiliza la prueba de la U de Mann -Whitney.

\section{Tabla 6}

Prueba de normalidad del Cuestionario de AECS

\begin{tabular}{ll}
\hline Prueba de normalidad & \\
\hline Kolmogorov-Smirnova & \\
Sig. & .000 \\
Escala Actitud Social & .000 \\
Escala Pensamiento Social
\end{tabular}

Fuente: elaboración propia

En la tabla 7, se muestra que en la escala Actitud Social existe diferencias entre las escalas según la variable sexo, mientras que para la escala de Pensamiento Social no existe diferencias por tanto se establecen diferentes normas de percentiles para cada escala $(\mathrm{p}<0.05)$. 


\section{Tabla 7}

Prueba de la U de Mann-Whitney de las escalas del cuestionario AECS según la variable sexo

\begin{tabular}{llcc}
\hline Hipótesis nula & Prueba & Sig. & Decisión \\
\hline Distribución de la escala & U de Mann -Whitney & .435 & $\begin{array}{c}\text { Rechaza } \\
\text { hipótesis } \\
\text { nula } \\
\text { Actitud Social es la misma }\end{array}$ \\
$\begin{array}{l}\text { entre las categorías de Sexo. } \\
\text { Distribución de la escala }\end{array}$ & U de Mann -Whitney & .831 & $\begin{array}{c}\text { Se acepta } \\
\text { hipótesis } \\
\text { nula }\end{array}$ \\
$\begin{array}{l}\text { Pensamiento Social es la } \\
\text { misma entre las categorías de }\end{array}$ & & & \\
Sexo. & & & \\
\hline
\end{tabular}

Fuente: elaboración propia

En la tabla 8 se aprecia los percentiles del cuestionario AECS, escala de Actitud Social en mujeres.

\section{Tabla 8}

Percentiles del cuestionario Actitudes y estrategias cognitivas sociales (AECS), escala de Actitud Social. Mujeres

\begin{tabular}{lcccccccccc}
\hline $\mathbf{P c}$ & CON & SEN & AC & LID & DOM & SF & AGR & AP & ANS & Pc \\
\hline $\mathbf{1}$ & 19 & 8 & 19 & 3 & 7 & 20 & 8 & 12 & 8 & $\mathbf{1}$ \\
$\mathbf{2}$ & 20 & 9 & 20 & 4 & 8 & 22 & 9 & 13 & 9 & $\mathbf{2}$ \\
$\mathbf{3}$ & 21 & 11 & 21 & 5 & 9 & 26 & 10 & 14 & 10 & $\mathbf{3}$ \\
$\mathbf{4}$ & 22 & 15 & 22 & 6 & 10 & 27 & 12 & 17 & 11 & $\mathbf{4}$ \\
$\mathbf{5}$ & 23 & 21 & 24 & 8 & 11 & 28 & 15 & 20 & 12 & $\mathbf{5}$ \\
$\mathbf{1 0}$ & 27 & 25 & 30 & 9 & 13 & 33 & 19 & 21 & 16 & $\mathbf{1 0}$ \\
$\mathbf{1 5}$ & 29 & 26 & 34 & 10 & 14 & 35 & 21 & 26 & 18 & $\mathbf{1 5}$ \\
$\mathbf{2 0}$ & 30 & 27 & 35 & 11 & 16 & 36 & 24 & 28 & 19 & $\mathbf{2 0}$ \\
$\mathbf{2 5}$ & 31 & 28 & 36 & 12 & 17 & 37 & 25 & 30 & 21 & $\mathbf{2 5}$ \\
$\mathbf{3 0}$ & 32 & 29 & 37 & 13 & 18 & 38 & 26 & 31 & 22 & $\mathbf{3 0}$ \\
$\mathbf{3 5}$ & 33 & 30 & 38 & 14 & 19 & 39 & 27 & 32 & 23 & $\mathbf{3 5}$ \\
$\mathbf{4 0}$ & 34 & 31 & 49 & 15 & 20 & 40 & 28 & 33 & 24 & $\mathbf{4 0}$ \\
$\mathbf{4 5}$ & 35 & 32 & 40 & 14 & 21 & 41 & 29 & 34 & 25 & $\mathbf{4 5}$ \\
$\mathbf{5 0}$ & 36 & 33 & 41 & 15 & 22 & 42 & 30 & 35 & 26 & $\mathbf{5 0}$ \\
$\mathbf{5 5}$ & 37 & 34 & 42 & 16 & 23 & 43 & 31 & 36 & 27 & $\mathbf{5 5}$ \\
$\mathbf{6 0}$ & 38 & 35 & 43 & 17 & 24 & 44 & 32 & 37 & 28 & $\mathbf{6 0}$ \\
$\mathbf{6 5}$ & 39 & 36 & 44 & 18 & 25 & 45 & 33 & 38 & 29 & $\mathbf{6 5}$
\end{tabular}




\begin{tabular}{ccccccccccc}
\hline Pc & CON & SEN & AC & LID & DOM & SF & AGR & AP & ANS & Pc \\
\hline $\mathbf{7 0}$ & 40 & 37 & 45 & 19 & 26 & 46 & 34 & 39 & 30 & $\mathbf{7 0}$ \\
$\mathbf{7 5}$ & 41 & 39 & 47 & 20 & 27 & 47 & 35 & 40 & 31 & $\mathbf{7 5}$ \\
$\mathbf{8 0}$ & 42 & 42 & 49 & 21 & 28 & 49 & 36 & 41 & 32 & $\mathbf{8 0}$ \\
$\mathbf{8 5}$ & 45 & 43 & 51 & 22 & 29 & 50 & 37 & 42 & 33 & $\mathbf{8 5}$ \\
$\mathbf{9 0}$ & 47 & 44 & 53 & 24 & 30 & 54 & 38 & 43 & 34 & $\mathbf{9 0}$ \\
$\mathbf{9 5}$ & 51 & 49 & 59 & 25 & 33 & 57 & 40 & 46 & 36 & $\mathbf{9 5}$ \\
$\mathbf{9 6}$ & 52 & 50 & 60 & 26 & 34 & 58 & 44 & 47 & 38 & $\mathbf{9 6}$ \\
$\mathbf{9 7}$ & 56 & 51 & 61 & 27 & 35 & 59 & 47 & 48 & 39 & $\mathbf{9 7}$ \\
$\mathbf{9 8}$ & 58 & 52 & 63 & 28 & 36 & 63 & 48 & 50 & 40 & $\mathbf{9 8}$ \\
$\mathbf{9 9}$ & 60 & 54 & 64 & 29 & 41 & 66 & 49 & 51 & 41 & $\mathbf{9 9}$ \\
\hline N & 423 & 423 & 423 & 423 & 423 & 423 & 423 & 423 & 423 & N \\
Media & 36.6 & 33.79 & 41.52 & 42.52 & 16.57 & 29.12 & 21.67 & 34.15 & 25.19 & Media \\
Mediana & 36.0 & 33.0 & 41.00 & 42.00 & 16.00 & 30.00 & 22.00 & 35.00 & 26.00 & Mediana \\
Mínimo & 14 & 8 & 14 & 20 & 4 & 8 & 7 & 12 & 8 & Mínimo \\
Máximo & 63 & 56 & 68 & 68 & 28 & 51 & 42 & 56 & 48 & Máximo \\
\hline
\end{tabular}

Fuente: elaboración propia

En la tabla 9 se aprecia los percentiles del cuestionario AECS, escala de Actitud Social en varones.

\section{Tabla 9}

Percentiles del cuestionario Actitudes y estrategias cognitivas sociales (AECS), escala de Actitud Social. Varones

\begin{tabular}{lcccccccccc}
\hline Pc & CON & SEN & AC & LID & DOM & SF & AGR & AP & ANS & Pc \\
\hline $\mathbf{1}$ & 14 & 8 & 16 & 19 & 4 & 8 & 7. & 12 & 9 & $\mathbf{1}$ \\
$\mathbf{2}$ & 19 & 14 & 19 & 20 & 6 & 12 & 8 & 13 & 10 & $\mathbf{2}$ \\
$\mathbf{3}$ & 20 & 15 & 20 & 21 & 7 & 13 & 9 & 19 & 11 & $\mathbf{3}$ \\
$\mathbf{4}$ & 21 & 19 & 21 & 22 & 8 & 15 & 10 & 20 & 12 & $\mathbf{4}$ \\
$\mathbf{5}$ & 24 & 22 & 24 & 23 & 9 & 16 & 11 & 21 & 13 & $\mathbf{5}$ \\
$\mathbf{1 0}$ & 29 & 25 & 31 & 31 & 10 & 19 & 13 & 25 & 16 & $\mathbf{1 0}$ \\
$\mathbf{1 5}$ & 30 & 27 & 35 & 35 & 11 & 21 & 14 & 26 & 18 & $\mathbf{1 5}$ \\
$\mathbf{2 0}$ & 31 & 28 & 36 & 37 & 12 & 23 & 15 & 29 & 19 & $\mathbf{2 0}$ \\
$\mathbf{2 5}$ & 32 & 29 & 37 & 38 & 13 & 24 & 16 & 30 & 21 & $\mathbf{2 5}$ \\
$\mathbf{3 0}$ & 33 & 30 & 38 & 39 & 14 & 25 & 17 & 31 & 22 & $\mathbf{3 0}$ \\
$\mathbf{3 5}$ & 34 & 31 & 39 & 40 & 15 & 26 & 18 & 32 & 23 & $\mathbf{3 5}$ \\
$\mathbf{4 0}$ & 35 & 32 & 40 & 41 & 16 & 27 & 19 & 33 & 24 & $\mathbf{4 0}$ \\
$\mathbf{4 5}$ & 36 & 33 & 41 & 42 & 17 & 28 & 20 & 34 & 25 & $\mathbf{4 5}$ \\
$\mathbf{5 0}$ & 37 & 34 & 42 & 43 & 18 & 29 & 21 & 35 & 26 & $\mathbf{5 0}$ \\
$\mathbf{5 5}$ & 38 & 35 & 43 & 44 & 19 & 30 & 22 & 36 & 27 & $\mathbf{5 5}$ \\
$\mathbf{6 0}$ & 39 & 36 & 44 & 45 & 20 & 31 & 23 & 37 & 28 & $\mathbf{6 0}$ \\
$\mathbf{6 5}$ & 40 & 37 & 45 & 46 & 21 & 32 & 24 & 38 & 29 & $\mathbf{6 5}$ \\
$\mathbf{7 0}$ & 41 & 38 & 46 & 47 & 22 & 33 & 25 & 39 & 30 & $\mathbf{7 0}$
\end{tabular}




\begin{tabular}{lcccccccccc}
$\mathbf{7 5}$ & 42 & 39 & 48 & 48 & 23 & 34 & 26 & 40 & 31. & $\mathbf{7 5}$ \\
$\mathbf{8 0}$ & 43 & 42 & 50 & 49 & 24 & 35 & 27 & 41 & 32 & $\mathbf{8 0}$ \\
$\mathbf{8 5}$ & 44 & 43 & 52 & 51 & 25 & 36 & 29 & 42 & 34 & $\mathbf{8 5}$ \\
$\mathbf{9 0}$ & 47 & 44 & 53 & 54 & 26 & 37 & 30 & 43 & 35 & $\mathbf{9 0}$ \\
$\mathbf{9 5}$ & 48 & 49 & 58 & 56 & 27 & 40 & 31 & 44 & 37 & $\mathbf{9 5}$ \\
$\mathbf{9 6}$ & 49 & 50 & 59 & 56 & 28 & 41 & 32 & 46 & 38 & $\mathbf{9 6}$ \\
$\mathbf{9 7}$ & 51 & 51 & 62 & 57 & 29 & 43 & 33 & 47 & 39 & $\mathbf{9 7}$ \\
$\mathbf{9 8}$ & 55 & 53 & 64 & 58 & 30 & 47 & 34 & 48 & 40 & $\mathbf{9 8}$ \\
$\mathbf{9 9}$ & 57 & 55 & 65 & 66 & 31 & 49 & 38 & 55 & 44.0 & $\mathbf{9 9}$ \\
& & & & & & & & & 0 & \\
\hline N & 556 & 556 & 556 & 556 & 556 & 556 & 556 & 556 & 556 & $\mathbf{N}$ \\
Media & 36.8 & 4.35 & 42.03 & 42.19 & 17.00 & 29.10 & 22.11 & 34.30 & 25.75 & Media \\
Mediana & 37.0 & 34.00 & 42 & 43.00 & 17.00 & 30.00 & 22.00 & 35.00 & 26.00 & Mediana \\
Mínimo & 10 & 8 & 14 & 19 & 4 & 8 & 7 & 12 & 8 & Mínimo \\
Máximo & 60 & 56 & 68 & 68 & 28 & 55 & 42 & 62 & 49 & Máximo \\
\hline
\end{tabular}

Fuente: elaboración propia

En la tabla 10 se aprecia los percentiles del cuestionario AECS, escala de Pensamiento Social en mujeres.

\section{Tabla 10}

Percentiles del cuestionario Actitudes y estrategias cognitivas sociales (AECS), escala de Pensamiento Social. Mujeres

\begin{tabular}{cccccccccccc}
\hline Pc & IMP & IND & CV & PER & DEM & HOS & OBS & ALT & CONS & MED & Pc \\
\hline $\mathbf{1}$ & 7 & 8 & 7 & 6 & 5 & 5 & 9 & 10 & 9 & 8 & $\mathbf{1}$ \\
$\mathbf{2}$ & 8 & 9 & 9 & 7 & 6 & 6 & 11 & 11 & 10 & 9 & $\mathbf{2}$ \\
$\mathbf{3}$ & 9 & 10 & 10 & 8 & 7 & 7 & 13 & 12 & 11 & 10 & $\mathbf{3}$ \\
$\mathbf{4}$ & 10 & 11 & 11 & 9 & 8 & 8 & 14 & 13 & 12 & 11 & $\mathbf{4}$ \\
$\mathbf{5}$ & 11 & 13 & 12 & 10 & 9 & 9 & 15 & 14 & 13 & 12 & $\mathbf{5}$ \\
$\mathbf{1 0}$ & 15 & 14 & 13 & 12 & 12 & 10 & 17 & 16 & 15 & 14 & $\mathbf{1 0}$ \\
$\mathbf{1 5}$ & 17 & 15 & 14 & 14 & 13 & 11 & 19 & 18 & 18 & 17 & $\mathbf{1 5}$ \\
$\mathbf{2 0}$ & 19 & 16 & 15 & 16 & 14 & 12 & 20 & 21 & 22 & 20 & $\mathbf{2 0}$ \\
$\mathbf{2 5}$ & 21 & 17 & 16 & 17 & 15 & 13 & 23 & 23 & 23 & 23 & $\mathbf{2 5}$ \\
$\mathbf{3 0}$ & 22 & 18 & 17 & 19 & 16 & 14 & 24 & 24 & 24 & 24 & $\mathbf{3 0}$ \\
$\mathbf{3 5}$ & 23 & 19 & 18 & 20 & 17 & 15 & 26 & 26 & 25 & 27 & $\mathbf{3 5}$ \\
$\mathbf{4 0}$ & 24 & 20 & 19 & 21 & 18 & 16 & 27 & 27 & 26 & 28 & $\mathbf{4 0}$ \\
$\mathbf{4 5}$ & 25 & 21 & 20 & 22 & 19 & 17 & 28 & 28 & 28 & 29 & $\mathbf{4 5}$ \\
$\mathbf{5 0}$ & 26 & 22 & 19 & 23 & 20 & 18 & 29 & 29 & 29 & 30 & $\mathbf{5 0}$ \\
$\mathbf{5 5}$ & 27 & 23 & 20 & 24 & 21 & 19 & 30 & 30 & 30 & 31 & $\mathbf{5 5}$ \\
$\mathbf{6 0}$ & 28 & 24 & 21 & 25 & 22 & 20 & 31 & 31 & 31 & 32 & $\mathbf{6 0}$ \\
$\mathbf{6 5}$ & 29 & 25 & 22 & 26 & 23 & 21 & 32 & 32 & 32 & 33 & $\mathbf{6 5}$ \\
$\mathbf{7 0}$ & 31 & 26 & 23 & 27 & 24 & 22 & 33 & 33 & 33 & 34 & $\mathbf{7 0}$ \\
$\mathbf{7 5}$ & 32 & 27 & 24 & 28 & 25 & 23 & 34 & 34 & 33 & 35 & $\mathbf{7 5}$ \\
$\mathbf{8 0}$ & 33 & 28 & 25 & 29 & 26 & 24 & 35 & 35 & 34 & 36 & $\mathbf{8 0}$ \\
$\mathbf{8 5}$ & 34 & 29 & 26 & 30 & 27 & 25 & 36 & 36 & 35 & 37 & $\mathbf{8 5}$ \\
$\mathbf{9 0}$ & 35 & 30 & 27 & 31 & 28 & 26 & 37 & 37 & 36 & 39 & $\mathbf{9 0}$ \\
$\mathbf{9 5}$ & 40 & 32 & 28 & 32 & 30 & 27 & 38 & 40 & 40 & 41 & $\mathbf{9 5}$ \\
$\mathbf{9 6}$ & 41 & 33 & 29 & 33 & 31 & 28 & 39 & 42 & 41 & 42 & $\mathbf{9 6}$ \\
$\mathbf{9 7}$ & 42 & 34 & 30 & 34 & 32 & 29 & 41 & 44 & 42 & 43 & $\mathbf{9 7}$ \\
$\mathbf{9 8}$ & 43 & 35 & 31 & 37 & 33 & 30 & 49 & 46 & 46 & 43 & $\mathbf{9 8}$
\end{tabular}




\begin{tabular}{cccccccccccc}
\hline Pc & IMP & \multicolumn{1}{c}{ IND } & CV & PER & \multicolumn{1}{c}{ DEM } & HOS & OBS & ALT & CONS & MED & Pc \\
\hline $\mathbf{9 9}$ & 45 & 40 & 32 & 41 & 34 & 32 & 54 & 49 & 54 & 53 & $\mathbf{9 9}$ \\
\hline $\mathbf{N}$ & 423 & 423 & 423 & 423 & 423 & 423 & 423 & 423 & 423 & 423 & $\mathbf{N}$ \\
Media & 25.36 & 21.53 & 19.1 & 21.80 & 19.77 & 16.93 & 28.10 & 28.13 & 27.65 & 28.22 & Media \\
Mediana & 26.0 & 22.0 & 20.0 & 23.0 & 20.0 & 19.0 & 29.0 & 30.0 & 29.00 & 30.0 & Mediana \\
Mínimo & 8 & 6 & 5 & 6 & 5 & 5 & 8 & 8 & 9 & 8 & Mínimo \\
Máximo & 49 & 42 & 35 & 42 & 35 & 35 & 55 & 53 & 56 & 56 & Máximo \\
\hline
\end{tabular}

Fuente: elaboración propia

En la tabla 11 se aprecia los percentiles del cuestionario AECS, escala de Pensamiento Social en varones.

\section{Tabla 11}

Percentiles del cuestionario Actitudes y estrategias cognitivas sociales (AECS), escala de Pensamiento Social. Varones

\begin{tabular}{lccccccccccc}
\hline Pc & IMP & IND & CV & PER & DEM & HOS & OBS & ALT & CONS & MED & Pc \\
\hline $\mathbf{1}$ & 8 & 6 & 7 & 6 & 5 & 5 & 10 & 10 & 9 & 8 & $\mathbf{1}$ \\
$\mathbf{2}$ & 9 & 8 & 8 & 7 & 6 & 6 & 11 & 11 & 10 & 9 & $\mathbf{2}$ \\
$\mathbf{3}$ & 10 & 9 & 9 & 8 & 7 & 7 & 12 & 12 & 11 & 10 & $\mathbf{3}$ \\
$\mathbf{4}$ & 11 & 10 & 10 & 9 & 8 & 8 & 13 & 13 & 12 & 11 & $\mathbf{4}$ \\
$\mathbf{5}$ & 12 & 11 & 11 & 10 & 10 & 9 & 14 & 14 & 13 & 12 & $\mathbf{5}$ \\
$\mathbf{1 0}$ & 15 & 12 & 12 & 12 & 11 & 10 & 17 & 15 & 14 & 13 & $\mathbf{1 0}$ \\
$\mathbf{1 5}$ & 17 & 14 & 13 & 14 & 13 & 11 & 18 & 16 & 16 & 16 & $\mathbf{1 5}$ \\
$\mathbf{2 0}$ & 19 & 15 & 14 & 16 & 15 & 12 & 20 & 20 & 19 & 19 & $\mathbf{2 0}$ \\
$\mathbf{2 5}$ & 21 & 17 & 15 & 17 & 16 & 13 & 22 & 23 & 23 & 20 & $\mathbf{2 5}$ \\
$\mathbf{3 0}$ & 22 & 18 & 16 & 18 & 17 & 14 & 23 & 24 & 24 & 23 & $\mathbf{3 0}$ \\
$\mathbf{3 5}$ & 23 & 19 & 17 & 19 & 18 & 15 & 24 & 26 & 25 & 26 & $\mathbf{3 5}$ \\
$\mathbf{4 0}$ & 24 & 20 & 18 & 20 & 19 & 16 & 26 & 27 & 26 & 27 & $\mathbf{4 0}$ \\
$\mathbf{4 5}$ & 25 & 21 & 19 & 21 & 20 & 17 & 27 & 28 & 27 & 28 & $\mathbf{4 5}$ \\
$\mathbf{5 0}$ & 26 & 22 & 20 & 21 & 21 & 18 & 28 & 29 & 28 & 20 & $\mathbf{5 0}$ \\
$\mathbf{5 5}$ & 27 & 23 & 21 & 22 & 22 & 19 & 29 & 30 & 29 & 30 & $\mathbf{5 5}$ \\
$\mathbf{6 0}$ & 28 & 24 & 22 & 23 & 23 & 20 & 30 & 31 & 30 & 31 & $\mathbf{6 0}$ \\
$\mathbf{6 5}$ & 29 & 25 & 23 & 24 & 24 & 21 & 31 & 32 & 31 & 32 & $\mathbf{6 5}$ \\
$\mathbf{7 0}$ & 30 & 26 & 24 & 25 & 25 & 22 & 32 & 33 & 32 & 33 & $\mathbf{7 0}$ \\
$\mathbf{7 5}$ & 31 & 27 & 25 & 26 & 26 & 23 & 33 & 34 & 33 & 34 & $\mathbf{7 5}$ \\
$\mathbf{8 0}$ & 32 & 28 & 26 & 27 & 27 & 24 & 34 & 35 & 34 & 35 & $\mathbf{8 0}$ \\
$\mathbf{8 5}$ & 33 & 29 & 27 & 28 & 28 & 25 & 35 & 36 & 35 & 37 & $\mathbf{8 5}$ \\
$\mathbf{9 0}$ & 34 & 30 & 28 & 29 & 29 & 26 & 38 & 37 & 36 & 38 & $\mathbf{9 0}$ \\
$\mathbf{9 5}$ & 39 & 31 & 29 & 32 & 30 & 27 & 39 & 40 & 39 & 41 & $\mathbf{9 5}$ \\
$\mathbf{9 6}$ & 40 & 32 & 30 & 33 & 31 & 28 & 40 & 42 & 40 & 42 & $\mathbf{9 6}$ \\
$\mathbf{9 7}$ & 41 & 33 & 31 & 34 & 32 & 29 & 42 & 43 & 41 & 43 & $\mathbf{9 7}$ \\
$\mathbf{9 8}$ & 44 & 34 & 32 & 37 & 33 & 30 & 48 & 44 & 47 & 44 & $\mathbf{9 8}$ \\
$\mathbf{9 9}$ & 45 & 35 & 33 & 39 & 34 & 33 & 53 & 51 & 53 & 53 & $\mathbf{9 9}$ \\
\hline N & 556 & 556 & 556 & 556 & 556 & 556 & 556 & 556 & 556 & 556 & $\mathbf{N}$ \\
Media & 25.4 & 21.5 & 19.4 & 21.85 & 19.62 & 17.62 & 27.62 & 28.07 & 26.96 & 27.5 & Media \\
Mediana & 26.0 & 23.0 & 20.0 & 22.00 & 20.00 & 19.00 & 28.00 & 30.00 & 28.00 & 30.0 & Mediana \\
Mínimo & 7 & 6 & 5 & 6 & 5 & 5 & 9 & 9 & 8 & 9 & Mínimo \\
Máximo & 47 & 41 & 33 & 42 & 35 & 35 & 56 & 56 & 56 & 56 & Máximo \\
\hline
\end{tabular}

Fuente: elaboración propia 


\section{Conclusiones}

La presente investigación fue demostrar que el instrumento posee adecuadas propiedades psicométricas. Se pudo trabajar la validez del constructo a través del análisis ítem y el índice de comunalidad donde la mayoría de los ítems presentan un nivel adecuado de correlación acorde al criterio establecido $\geq 0.20$, sin embargo, hay nueve ítems solo en la escala de actitudes sociales que están por debajo de este criterio sin embargo con respecto a la comunalidad solo se encontró un ítem que no cumple con lo criterio establecido $\geq 0.30$.

Respecto a la confiabilidad de las escalas de actitudes sociales oscilan entre 0.481 y 0.741, indicando que las escalas de Sensibilidad Social (Sen), Ayuda y colaboración (Ac), Seguridad y firmeza (Sf), Liderazgo prosocial (Lid), Dominancia (Dom), Ansiedad-timidez (Ans) y Agresividad-terquedad (Agr) alcanzaron niveles de confiabilidad alto; no obstante, Conformidad (Con) y Apatía-retraimiento (Ap) se encuentran en el nivel medio (Palella y Martins, 2012). En tanto que para las escalas de Pensamiento social se observa que los valores de confiabilidad oscilan entre 0.612 y 0.827 , indicando que todas las escalas alcanzaron niveles de confiabilidad alto. (Palella y Martins, 2012). Estos datos son parecidos a los encontrados por Castillo (2014) en adolescentes donde encontraron en la escala de Actitud social valores entre .487 y .714 y en la escala de Pensamiento Social .430 y .718; en tanto Garaigordobil y Peña (2014) quienes realizaron un experimento cuasi experimental utilizando solo la escala de Actitud social cuyos valores oscilan entre .550 y .770 .

En cuanto al objetivo está enfocado a determinar las normas en percentiles según el sexo, para ello, se determinó primero que la prueba de K-S es de distribución no paramétrica, por ello, se utilizó la prueba de U Man- Whitney encontrándose diferencias significativas entre mujeres y varones, encontrándose que existen diferencias significativas $\mathrm{p}<0.05$ estableciendo baremos para cada escala según el sexo. Tales resultados son semejantes a los encontrados por Moraleda et al. (2004), quienes establecieron normas en percentiles según la variable sexo basándose en el análisis de diferencias significativas $(\mathrm{p}<0.05)$.

Conclusiones:

Se concluye que el instrumento AESC cuenta con una validez adecuada ya que en el análisis del KMO tuvo (.827) la cual indica que es aceptable para el análisis factorial ya que mientras esté más cerca al 1 es mejor. Asimismo, en la prueba de esfericidad de Bartlett (.000) indica, es menor que 0.05 la cual es indicado el análisis factorial. Por otro lado, en el análisis de comunalidades casi su totalidad de los ítems de ambas escalas superan el $\geq 0.30$ que es el criterio establecido.

Con respecto a su confiabilidad de las escalas de actitud social oscilan entre $0.481 \mathrm{y}$ 0.741. Asimismo, confiabilidad de las escalas de pensamiento social oscilan entre 0.612 y 0.827 . En resumen, se puede certificar que el cuestionario AESC cuenta con la base teórica y psicométrica competente para ser empleado en la medición del constructo en adolescentes. 


\section{Referencias bibliográficas}

Betina, A. y Contini, N. (2011). Las habilidades sociales en niños y adolescentes. Su importancia en la prevención de trastornos psicopatológicos. Fundamentos en Humanidades. 1, 159 182.

Bravo, I. y Herrera, L. (2011). Convivencia escolar en educación primaria. Las habilidades sociales del alumnado como variable moduladora. Dedica Revista de educación y humanidades, $1,173-212$.

Castillo, C. (2014). Propiedades psicométricas del cuestionario de actitudes y estrategias cognitivas sociales en adolescentes del nivel secundario de la provincia de Chepén (Tesis de Licenciatura), Universidad César Vallejo, Trujillo, Perú.

Castillo, M. y Eugenio, D. (2019). Actitudes y estrategias cognitivas sociales en adolescentes de colegios público y privado del distrito de José Luis Bustamante y Rivero-Arequipa (Tesis de Licenciatura), Universidad Nacional de San Agustín, Arequipa, Perú.

Cholán, V. (2018). Actitudes y estrategias cognitivas sociales en Estudiantes de secundaria del tercio superior E inferiores de una institución educativa Nacional del distrito de Moche (Tesis de Maestría), Universidad Privada del Norte, Trujillo, Perú.

Del Prette, A., Del Prette, Z. y Mendes M. (1999). Habilidades Sociales en la Formación Profesional del Psicólogo: Análisis de un programa de Intervención. Psicología Conductual, Universidad Federal de São Carlos, Brasil, 7 (1), 27-47

Del Prette. Z.A.P. y Del Prette, A. (2002a). Psicología das habilidades sociais: terapia e educação. Petrópolis - RJ: Editora Vozes.

Fernández, O., Luquez, P. y Leal, E. (2010). Procesos socioafectivos asociados al aprendizaje y práctica de valores en el ámbito escolar Telos, vol. 12, (1), 63-78.

Garaigordobil, M y Peña, A (2014). Intervención en las habilidades sociales: efectos en la inteligencia emocional y la conducta social. Behavioral Psychology / Psicología Conductual, 22, (3), 551-567

García, C. (2004). La medición en Ciencias sociales y en la Psicología: Estadística con SPSS y metodología de la investigación. México: Trillas

García, A.D. (2010). Estudio sobre el asertividad y las habilidades sociales en el alumnado de educación social. XXI Revista de Educación, 12, pp. 225-239.

Hernández, R., Fernández, C., y Baptista, P. (2014). Metodología de la Investigación. México: Mc Graw Hill.

López, M; Garrido, V.; Rodríguez, F. y Paíno, S. (2002). Jóvenes y competencia social: Un programa de intervención. Psicothema, 14, 155-162.

Lorente, S. (2014). Efecto de la competencia social, la empatía y la conducta prosocial en adolescentes. (Tesis Doctoral). Universidad de Valencia. España

Moraleda, M., González, A., y García Gallo, J. (2004). Actitudes y Estrategias Cognitivas Sociales. Madrid: TEA Ediciones.

Roca, M. (2003). Inteligencia emocional: ¿Qué es y cómo desarrollarla? Trabajo presentado en el Seminario «Educando para la Responsabilidad Social: La universidad en su función docente», mayo, Santiago de Chile.

Rodríguez y Morelio (2012). Validación de instrumentos psicológicos: Criterios básicos. Cuba: Editorial Feijóo.

Salovey, P. y Mayer J. D. (1990). Emotional Intelligence. Imagination, Cognition and Personality, SAGE journals, 9, 185-211.

Esta obra se comparte bajo la licencia Creative Common Atribución-No Comercial 4.0 International (CC BY-NC 4.0) Revista de la Universidad Internacional del Ecuador. URL: https://www.uide.edu.ec/ 
Méndez, G., Guamán, M., Siguenza, W. G., y Espinoza, A. (2018). Estudio descriptivo de los sucesos de vida estresores en adolescentes. INNOVA Research Journal, 3(6), 40-52. https://doi.org/10.33890/innova.v3.n6.2018.547

Monjas Casares, M. I. (1993) - Programa de Enseñanza de Habilidades de Interacción Social (PEHIS) para niños y niños en edad escolar. (1 ed.) Valladolid: Casares ImpresionesAS (Trilce).

Moraleda, M. (1998). Educar en la Competencia Social. Un programa para la tutoría para adolescentes. Madrid: CCS

Moraleda, M., González, A., y García Gallo, J. (2004). Actitudes y Estrategias Cognitivas Sociales. Madrid: TEA Ediciones.

Moraleda, M., Oñate, M.P. y González, E. (1999). Educar en la Competencia Social - un programa para la tutoría con adolescentes. Madrid: Editorial CCS.

Palella, S., y Martins F. (2008). Metodología de la Investigación Cuantitativa. Caracas: Fedupel.

Plunkett, S., Radmacher, K. Kimberly, A. y Moll-Phanara, D. (2000). Adolescent life events, stress and coping: A comparison of communities and genders. Professional School Counseling, 3,5, 356-367

Rangel, P. (2015). Relación entre dinámica familiar y competencia social del niño preescolar de 3 a 5 años. (Tesis para el grado de magister). Lima, Perú.

Ruiz, J. (2017). Estrategia metodológica basada en la teoría de Bandura para desarrollar actitudes positivas hacia el área de ciencia y ambiente en los estudiantes del tercer ciclo de la Institución Educativa los Embajadores del saber. (Tesis de maestría) Universidad Nacional del Santa, Chimbote, Perú.

Santos, O. (2015). Adaptación del cuestionario sobre actitudes y estrategias cognitivas sociales (A.E.C.S) a la población brasileña. (Tesis Doctoral). Universidad Complutense De Madrid, Madrid, España.

Trianes, M., Muñoz, M. y Jiménes, M., (2007) Las relaciones sociales en la infancia y en la adolescencia y sus problemas. Madrid: Pirámide.

Perinat, A. (2007). Psicología del desarrollo un enfoque sistemático. España: Editorial UOC. 